The Journal of Nonlinear $\mathbf{S}$ ciences and Applications

http://www.tjnsa.com

\title{
THE KANNAN'S FIXED POINT THEOREM IN A CONE RECTANGULAR METRIC SPACE
}

\author{
MOHAMED JLELI ${ }^{1}$ AND BESSEM SAMET ${ }^{2 *}$ \\ Communicated by I. Beg
}

\begin{abstract}
Recently, Azam, Arshad and Beg introduced the notion of cone rectangular metric spaces by replacing the triangular inequality of a cone metric space by a rectangular inequality. In this paper, we extend the Kannan's fixed point theorem in such spaces.
\end{abstract}

\section{INTRODUCTION}

If $(X, d)$ is a complete metric space and $T: X \rightarrow X$ is a contraction, i.e.,

$$
d(T x, T y) \leq \alpha d(x, y)
$$

for all $x, y \in X$ with $\alpha \in[0,1)$, then the widely known Banach's contraction mapping principle tells that $T$ has a unique fixed point in $X$. A lot of generalizations of this theorem have been done, mostly by relaxing the contraction condition and sometimes by withdrawing the requirement of completeness or even both [4, 5, 6, 7, 10, 11, 12, 13.

Huang and Zhang [8] have introduced the concept of cone metric space, where the set of real numbers is replaced by an ordered Banach space, and they have established some fixed point theorems for contractive type mappings in a normal cone metric space. The study of fixed point theorems in such spaces is followed by some other mathematicians, see [1, 9].

Following the idea of Branciari [3], Azam, Arshad and Beg [2] extended the notion of cone metric spaces by replacing the triangular inequality by a rectangular inequality. The aim of this paper is to extend the Kannan's fixed point theorem

Date: Received: 10 April 2009 ; Revised 07 June 2009.

* Corresponding author.

2000 Mathematics Subject Classification. Primary 47H10; Secondary 54E35, 54E50.

Key words and phrases. Cone rectangular metric space; Kannan's fixed point theorem. 
[11] in such spaces. We start by recalling some definitions introduced in [2, 8] and preliminary results.

Let $E$ always be a real Banach space and $P$ a subset of $E$. $P$ is called a cone if and only if:

(i) $P$ is closed, nonempty, and $P \neq\{0\}$.

(ii) $a, b \in \mathbb{R}, a, b \geq 0, x, y \in P \Rightarrow a x+b y \in P$.

(iii) $x \in P$ and $-x \in P \Rightarrow x=0$.

Given a cone $P \subset E$, we define a partial ordering $\leq$ with respect to $P$ by:

$$
x \leq y \Leftrightarrow y-x \in P .
$$

We shall write $x<y$ to indicate that $x \leq y$ but $x \neq y$, while $x \ll y$ will stand for $y-x \in \operatorname{int} P$, int $P$ denotes the interior of $P$.

The cone $P$ is called normal if there is a number $k>0$ such that for all $x, y \in E$,

$$
0 \leq x \leq y \Rightarrow\|x\| \leq k\|y\|
$$

where $\|\cdot\|$ is the norm in $E$. In this case, the number $k$ is called the normal constant of $P$.

In the following we always suppose $E$ is a Banach space, $P$ is a cone in $E$ with int $P \neq \emptyset$ and $\leq$ is partial ordering with respect to $P$.

Definition 1.1. Let $X$ be a nonempty set. Suppose the mapping $d: X \times X \rightarrow E$ satisfies:

(a) $0<d(x, y)$ for all $x, y \in X, x \neq y$ and $d(x, y)=0$ if and only if $x=y$.

(b) $d(x, y)=d(y, x)$ for all $x, y \in X$.

(c) $d(x, y) \leq d(x, u)+d(u, v)+d(v, y)$ for all $x, y \in X$ and for all distinct points $u, v \in X \backslash\{x, y\}$ (rectangular property).

Then $d$ is called a cone rectangular metric on $X$, and $(X, d)$ is called a cone rectangular metric space.

Not that any cone metric space is a cone rectangular metric space but the converse is not true in general.

Example 1.2. Let $E=\mathbb{R}^{2}, P=\{(x, y) \in E \mid x, y \geq 0\}, X=\mathbb{R}, d: X \times X \rightarrow E$ such that

$$
d(x, y)= \begin{cases}(0,0) & \text { if } \quad x=y, \\ (3 \alpha, 3) & \text { if } x \text { and } y \text { are in }\{1,2\}, x \neq y, \\ (\alpha, 1) & \text { if } x \text { and } y \text { can not both at a time in }\{1,2\}, x \neq y,\end{cases}
$$

where $\alpha>0$ is a constant. Then $(X, d)$ is a cone rectangular metric space but it is not a cone metric space since we have $d(1,2)=(3 \alpha, 3)>d(1,3)+d(3,2)=(2 \alpha, 2)$.

Example 1.3. Let $E=\mathbb{R}^{2}, P=\{(x, y) \in E \mid x, y \geq 0\}, X=\{a, b, c, e\}$ and $d: X \times X \rightarrow E$ such that

$$
\left\{\begin{array}{l}
d(x, x)=(0,0), \forall x \in X, \\
d(x, y)=d(y, x), \forall x, y \in X, \\
d(a, b)=(3, \alpha), \\
d(a, c)=d(b, c)=(1, \alpha), \\
d(a, e)=d(b, e)=d(c, e)=(2, \alpha),
\end{array}\right.
$$


where $\alpha>0$ is a constant. Then $(X, d)$ is a cone rectangular metric space but it is not a cone metric space since we have $d(a, b)=(3, \alpha)$ and $d(a, c)+d(c, b)=(2,2 \alpha)$ but $(3, \alpha)$ and $(2,2 \alpha)$ cannot be compared with respect to $\leq$.

Definition 1.4. Let $(X, d)$ be a cone rectangular metric space. Let $\left(x_{n}\right)$ be a sequence in $X$ and $x \in X$. If for every $c \in E, c \gg 0$ there is $N$ such that for all $n>N, d\left(x_{n}, x\right) \ll c$, then $\left(x_{n}\right)$ is said to be convergent to $x$ and $x$ is the limit of $\left(x_{n}\right)$. We denote this by $x_{n} \rightarrow x$ as $n \rightarrow+\infty$.

The proof of this result is identical to the proof of ([8]-Lemma 1).

Lemma 1.5. Let $(X, d)$ be a cone rectangular metric space, $P$ be a normal cone. Let $\left(x_{n}\right)$ be a sequence in $X$. Then,

$$
x_{n} \rightarrow x \text { as } n \rightarrow+\infty \Leftrightarrow\left\|d\left(x_{n}, x\right)\right\| \rightarrow 0 \text { as } n \rightarrow+\infty .
$$

Note that if $(X, d)$ is a cone metric space and $\left(x_{n}\right)$ is a convergent sequence in $X$, then the limit of $\left(x_{n}\right)$ is unique ([8]-Lemma 2$)$. In our case, the uniqueness of the limit is not satisfied in general. We give an example to illustrate this remark.

Example 1.6. We take $E=\mathbb{R}$ and $P=\{x \in \mathbb{R} \mid x \geq 0\}$. Let $\left(x_{n}\right)_{n \in \mathbb{N}^{*}}$ be a sequence in $\mathbb{Q}$ and $a, b \in \mathbb{R} \backslash \mathbb{Q}, a \neq b$. We put $X=\left\{x_{1}, x_{2}, \cdots, x_{n}, \cdots\right\} \cup\{a, b\}$ and we consider $d: X \times X \rightarrow \mathbb{R}$ defined by

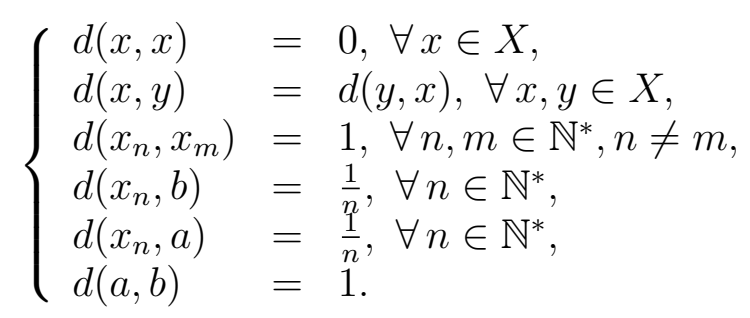

We remark that $(X, d)$ is not a cone metric space because we have

$$
d\left(x_{2}, x_{3}\right)=1>d\left(x_{2}, a\right)+d\left(a, x_{3}\right)=\frac{1}{2}+\frac{1}{3}=\frac{5}{6} .
$$

However, $(X, d)$ is a cone rectangular metric space. Now, since $d\left(x_{n}, a\right)=\frac{1}{n} \rightarrow 0$ as $n \rightarrow+\infty$, we obtain that $x_{n} \rightarrow a$ as $n \rightarrow+\infty$. Also, we have $d\left(x_{n}, b\right)=\frac{1}{n} \rightarrow 0$ as $n \rightarrow+\infty$ and then $x_{n} \rightarrow b$ as $n \rightarrow+\infty$.

Definition 1.7. Let $(X, d)$ be a cone rectangular metric space, $\left(x_{n}\right)$ be a sequence in X. If for any $c \in E$ with $0 \ll c$, there is $N$ such that for all $n, m>N$, $d\left(x_{n}, x_{m}\right) \ll c$, then $\left(x_{n}\right)$ is called a Cauchy sequence in $X$.

The proof of the following result is similar to the proof of ([8]-Lemma 4).

Lemma 1.8. Let $(X, d)$ be a cone rectangular metric space and $P$ be a normal cone. Let $\left(x_{n}\right)$ be a sequence in $X$. Then $\left(x_{n}\right)$ is a Cauchy sequence if and only if $d\left(x_{n}, x_{m}\right) \rightarrow 0$ as $n, m \rightarrow+\infty$.

Note that if $(X, d)$ is a cone metric space and $\left(x_{n}\right)$ is a convergent sequence in $X$, then $\left(x_{n}\right)$ is a Cauchy sequence ([8]-Lemma 3$)$. In our case, this result is not true in general. In fact, in Example 1.6, the sequence $\left(x_{n}\right)$ is convergent but we have $d\left(x_{n}, x_{m}\right) \rightarrow 1$ as $n, m \rightarrow+\infty$. 
Definition 1.9. Let $(X, d)$ be a cone rectangular metric space. If every Cauchy sequence is convergent in $X$, then $X$ is called a complete cone rectangular metric space.

In this particular case, the uniqueness of the limit is satisfied.

Lemma 1.10. Let $(X, d)$ be a complete cone rectangular metric space, $P$ be a normal cone with normal constant $k$. Let $\left(x_{n}\right)$ be a Cauchy sequence in $X$ and suppose that there is $N$ such that

(i) $x_{n} \neq x_{m}$ for all $n, m>N$.

(ii) $x_{n}, x$ are distinct points in $X$ for all $n>N$.

(iii) $x_{n}, y$ are distinct points in $X$ for all $n>N$.

(iii) $x_{n} \rightarrow x$ and $x_{n} \rightarrow y$ as $n \rightarrow+\infty$.

Then $x=y$.

Proof. For any $c \in E$ with $0 \ll c$, there is $\nu$ such that

$$
d\left(x_{n}, x\right) \ll c, \quad d\left(x_{n}, y\right) \ll c \quad \text { and } \quad d\left(x_{n}, x_{m}\right) \ll c
$$

for all $n, m>\nu$. For all $n, m>\max (N, \nu)$, We have

$$
d(x, y) \leq d\left(x, x_{n}\right)+d\left(x_{n}, x_{m}\right)+d\left(x_{m}, y\right) \leq 3 c .
$$

Hence, $\|d(x, y)\| \leq 3 k\|c\|$. Since $c$ is arbitrary $d(x, y)=0$; therefore $x=y$.

\section{MAIN RESULT}

In this section, we derive a fixed point theorem in a cone rectangular metric space. Our obtained result generalizes the well known Kannan's theorem.

Theorem 2.1. Let $(X, d)$ be a complete cone rectangular metric space, $P$ be a normal cone with normal constant $k$. Suppose a mapping $T: X \rightarrow X$ satisfies the contractive condition

$$
d(T x, T y) \leq \alpha(d(T x, x)+d(T y, y)), \forall x, y \in X,
$$

where $\alpha \in[0,1 / 2)$. Then,

(i) $T$ has a unique fixed point in $X$.

(ii) For any $x \in X$, the iterative sequence $\left(T^{n} x\right)$ converges to the fixed point.

Proof. Let $x \in X$. We have

$$
d\left(T x, T^{2} x\right) \leq \alpha\left(d(T x, x)+d\left(T x, T^{2} x\right)\right),
$$

i.e.,

Again

$$
d\left(T x, T^{2} x\right) \leq \frac{\alpha}{1-\alpha} d(x, T x) .
$$

$$
d\left(T^{2} x, T^{3} x\right) \leq \alpha\left(d\left(T x, T^{2} x\right)+d\left(T^{2} x, T^{3} x\right)\right)
$$

i.e.,

$$
d\left(T^{2} x, T^{3} x\right) \leq \frac{\alpha}{1-\alpha} d\left(T x, T^{2} x\right) \leq\left(\frac{\alpha}{1-\alpha}\right)^{2} d(x, T x) .
$$


Thus in general, if $n$ is a positive integer, then

$$
d\left(T^{n} x, T^{n+1} x\right) \leq\left(\frac{\alpha}{1-\alpha}\right)^{n} d(x, T x)=r^{n} d(x, T x),
$$

where $r=\frac{\alpha}{1-\alpha} \in[0,1)$.

We divide the proof into two cases.

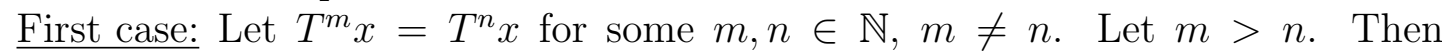
$\overline{T^{m-n}\left(T^{n} x\right)}=T^{n} x$, i.e. $T^{p} y=y$ where $p=m-n, y=T^{n} x$. Now since $p>1$, we have

$$
\begin{aligned}
d(y, T y) & =d\left(T^{p} y, T^{p+1} y\right) \\
& \leq r^{p} d(y, T y) .
\end{aligned}
$$

Since $r \in[0,1)$, we obtain $-d(y, T y) \in P$ and $d(y, T y) \in P$ which implies that $\|d(y, T y)\|=0$, i.e., $T y=y$.

Second case: Assume that $T^{m} x \neq T^{n} x$ for all $m, n \in \mathbb{N}, m \neq n$. Clearly, we have

$$
d\left(T^{n} x, T^{n+1} x\right) \leq r^{n} d(x, T x) \leq \frac{r^{n}}{1-r} d(x, T x)
$$

and

$$
\begin{aligned}
d\left(T^{n} x, T^{n+2} x\right) & \leq \alpha\left(d\left(T^{n-1} x, T^{n} x\right)+d\left(T^{n+1} x, T^{n+2} x\right)\right) \\
& \leq \alpha\left(r^{n-1} d(x, T x)+r^{n+1} d(x, T x)\right) \\
& \leq r^{n} d(x, T x)+r^{n+1} d(x, T x) \\
& \leq \frac{r^{n}}{1-r} d(x, T x) .
\end{aligned}
$$

Now if $m>2$ is odd then writing $m=2 \ell+1, \ell \geq 1$ and using the fact that $T^{p} x \neq T^{r} x$ for $p, r \in \mathbb{N}, p \neq r$, we can easily show that

$$
\begin{aligned}
d\left(T^{n} x, T^{n+m} x\right) & \leq d\left(T^{n} x, T^{n+1} x\right)+d\left(T^{n+1} x, T^{n+2} x\right)+\cdots+d\left(T^{n+2 \ell} x, T^{n+2 \ell+1} x\right) \\
& \leq r^{n} d(x, T x)+r^{n+1} d(x, T x)+\cdots+r^{n+2 \ell} d(x, T x) \\
& \leq \frac{r^{n}}{1-r} d(x, T x) .
\end{aligned}
$$

Again if $m>2$ is even then writing $m=2 \ell, \ell \geq 2$ and using the same arguments as before, we can get

$$
\begin{aligned}
& d\left(T^{n} x, T^{n+m} x\right) \\
\leq & d\left(T^{n} x, T^{n+2} x\right)+d\left(T^{n+2} x, T^{n+3} x\right)+d\left(T^{n+3} x, T^{n+4} x\right)+\cdots+d\left(T^{n+2 \ell-1} x, T^{n+2 \ell} x\right) \\
\leq & r^{n} d(x, T x)+r^{n+2} d(x, T x)+r^{n+3} d(x, T x)+\cdots+r^{n+2 \ell-1} d(x, T x) \\
\leq & \frac{r^{n}}{1-r} d(x, T x) .
\end{aligned}
$$

Thus combining all the cases we have

$$
d\left(T^{n} x, T^{n+m} x\right) \leq \frac{r^{n}}{1-r} d(x, T x), \forall m, n \in \mathbb{N} .
$$


Hence, we get

$$
\left\|d\left(T^{n} x, T^{n+m} x\right)\right\| \leq k \frac{r^{n}}{1-r}\|d(x, T x)\|, \forall m, n \in \mathbb{N} .
$$

Since $k \frac{r^{n}}{1-r}\|d(x, T x)\| \rightarrow 0$ as $n \rightarrow+\infty,\left(T^{n} x\right)$ is a Cauchy sequence. By the completeness of $X$, there is $x^{*} \in X$ such that $T^{n} x \rightarrow x^{*}$ as $n \rightarrow+\infty$.

We shall now show that $T x^{*}=x^{*}$. Without any loss of generality, we can assume that $T^{r} x \neq x^{*}, T x^{*}$ for any $r \in \mathbb{N}$. We have

$$
\begin{aligned}
d\left(x^{*}, T x^{*}\right) & \leq d\left(x^{*}, T^{n} x\right)+d\left(T^{n} x, T^{n+1} x\right)+d\left(T^{n+1} x, T x^{*}\right) \\
& \leq d\left(x^{*}, T^{n} x\right)+d\left(T^{n} x, T^{n+1} x\right)+\alpha\left(d\left(T^{n} x, T^{n+1} x\right)+d\left(x^{*}, T x^{*}\right)\right)
\end{aligned}
$$

which implies that

$$
d\left(x^{*}, T x^{*}\right) \leq \frac{1}{1-\alpha}\left(d\left(x^{*}, T^{n} x\right)+(1+\alpha) d\left(T^{n} x, T^{n+1} x\right)\right) .
$$

Hence,

$\left\|d\left(x^{*}, T x^{*}\right)\right\| \leq \frac{k}{1-\alpha}\left(\left\|d\left(x^{*}, T^{n} x\right)\right\|+(1+\alpha)\left\|d\left(T^{n} x, T^{n+1} x\right)\right\|\right) \rightarrow 0$ as $n \rightarrow+\infty$.

So we obtain $d\left(T x^{*}, x^{*}\right)=0$, i.e., $x^{*}=T x^{*}$.

Now, if $y^{*}$ is another fixed point of $T$, then

$$
d\left(x^{*}, y^{*}\right)=d\left(T x^{*}, T y^{*}\right) \leq \alpha\left(d\left(x^{*}, T x^{*}\right)+d\left(y^{*}, T y^{*}\right)\right)=0
$$

which implies that $\left\|d\left(x^{*}, y^{*}\right)\right\|=0$, i.e., $x^{*}=y^{*}$.

To illustrate Theorem 2.1, we give the following example.

Example 2.2. Let $E=\mathbb{C}$ and $P=\{x+i y \mid x, y \in \mathbb{R}, x, y \geq 0\}$ a normal cone in $E$. Let $X=\{1,2,3,4\}$. Define $d: X \times X \rightarrow E$ by

$$
\begin{aligned}
& d(x, x)=0 \\
& d(1,2)=d(2,1)=3+9 i \\
& d(2,3)=d(3,2)=d(1,3)=d(3,1)=1+3 i \\
& d(1,4)=d(4,1)=d(2,4)=d(4,2)=d(3,4)=d(4,3)=4+12 i .
\end{aligned}
$$

Then $(X, d)$ is a complete cone rectangular metric space but $(X, d)$ is not a cone metric space because it lacks the triangular property

$$
3+9 i=d(1,2)>d(1,3)+d(3,2)=2+6 i .
$$

Now, define a mapping $T: X \rightarrow X$ as follows

$$
T x=\left\{\begin{array}{lll}
3 & \text { if } & x \neq 4 \\
1 & \text { if } & x=4
\end{array}\right.
$$

We remark that $T$ is not a contractive mapping with respect to the standard metric in $X$ because we have

$$
|T 4-T 2|=2=|4-2| .
$$

However, $T$ satisfies

$$
d(T x, T y) \leq \alpha(d(x, T x)+d(y, T y)), \forall x, y \in X
$$


with $\alpha=\frac{1}{3}$. Applying Theorem 2.1, we obtain that $T$ admits a unique fixed point, that is $x^{*}=3$.

Note that in this example, results of Huang and Zhang [8] are not applicable to obtain the fixed point of the mapping $T$ on $X$, since $(X, d)$ is not a cone metric space.

Acknowledgements: The authors would like to thank Professor Ismat Beg for providing them with the reference [2].

\section{REFERENCES}

1. M. Abbas and G. Jungck, Common fixed point results for noncommuting mappings without continuity in cone metric spaces, J. Math. Anal. Appl, 341 (2008), 416-420. 1

2. A. Azam, M. Arshad and I. Beg, Banach contraction principle on cone rectangular metric spaces, Appl. Anal. Discrete Math, (to appear). 1, 2

3. A. Branciari, A fixed point theorem of Banach-Caccioppoli type on a class of generalized metric spaces, Publ. Math. Debrecen, 57 1-2 (2000), 31-37. 1

4. H. Chatterjee, On generalization of Banach Contraction Principle, Indian J. Pure appl. Math, 10 (1979), 400-403. 1

5. Lj. B. Ciric, On contraction type mappings, Math. Balkanica, 1 (1971), 52-57. 1

6. Lj. B. Ciric, A generalization of Banach's contraction principle, Proc. Am. Math. Soc, 45 (1974), 267-273. 1

7. B. K. Dass and S. Gupta, An extension of Banach contraction principle through rational expression, Indian J. Pure appl. Math, 6 (1975), 1455-1458. 1

8. L.G. Huang and X. Zhang, Cone metric spaces and fixed point theorems of contractive mappings. J. Math. Anal. Appl, 332 (2007), 1468-1476. 1, 1, 1, 1, 1, 2.2

9. D. Ilic and V. Rakocevic, Common fixed points for maps on cone metric space, J. Math. Anal. Appl, 341 (2008), 876-882. 1

10. R. Kannan, Some results on fixed points, Bull. Cal. Math. Soc, (60) 1-2 (1968), 71-76. 1

11. R. Kannan, Some results on fixed points-II. Amer. Math. Monthly, (76)(1969), 405-408. 1

12. D. Mihet, A counterexample to "Common fixed point theorem in probabilistic quasi-metric spaces", J. Nonlinear Sci. Appl. 1 (2008), no. 2, 121-122. 1

13. A. R. Shabani and S. Ghasempour, Common fixed point theorem in probabilistic quasimetric spaces, J. Nonlinear Sci. Appl. 1 (2008), no. 1, 31-35. 1

${ }^{1}$ Department of Mathematics, Tunis College of Sciences and Techniques, 5 Avenue Taha Hussein, BP, 56, Bab Manara, Tunis.

E-mail address: jlelimohamed@gmail.com

${ }^{2}$ Department of Mathematics, Tunis College of Sciences and Techniques, 5 Avenue Taha Hussein, BP, 56, Bab Manara, Tunis.

E-mail address: bessem.samet@gmail.com 